\title{
Originalartikel
}

\section{Verteilung von Varroa jacobsoni im drohnenfreien Bienenvolk (Apis mellifera carnica)}

\author{
J Steiner \\ Zoologisches Institut, LS Entwicklungsphysiologie, Universität Tübingen, \\ Auf der Morgenstelle 28, D-7400 Tübingen, Deutschland
}

(Eingegangen 4 August 1992, angenommen 3 November 1992)

Zusammenfassung - In einem Herbstvolk von Apis mellifera carnica wurde beobachtet, daß die Milbenweibchen nach dem Schlüpfen größtenteils ihre Trägerbienen sogleich wechseln. Im Alter bis zu 3 Tagen waren durchschnittlich 15\%, ab 6 Tagen 30\% der Bienen befallen. Mögliche Ursachen für diese Verteilung werden diskutiert.

\section{Varroa jacobsoni / Apis mellifera carnica / Parasitierungsverhalten / Wirtswahl}

\section{EINLEITUNG}

Mit dem Auslaufen von Bienenbrut werden in Varroatose-Völkern adulte Milbenweibchen freigesetzt, und zwar sowohl Mutter- wie Tochtermilben. Je nach Parasitierungsgrad der einzelnen Brutzelle und Reproduktionserfolg der Varroa-Weibchen schlüpfen zusammen mit einer Jungbiene eine oder mehrere adulte Milben aus. Verbleiben sie auf "ihrer" Jungbiene, oder suchen sie sich andere Trägerbienen aus? Zu dieser Frage gibt es kaum Daten (Ifantidis und Rosenkranz, 1988). In Käfigversuchen wiesen Le Conte und Arnold (1987) nach, daß Milben, die auf frisch geschlüpfte Bienen gesetzt worden waren, rasch auf mindestens 2 Tage ältere überwechselten. Eine auffällig geringe Attraktivität frisch geschlüpfter Bienen für Milben beobachteten auch Kraus et al (1986) in Arena-Versuchen. Es wurde außerdem mehrfach beschrieben, daß die Milben während der Fortpflanzungssaison der Bienen als Träger Ammen ebenso wie Stockdrohnen bevorzugen (Review: Ritter, 1981). Für die Unterscheidung junger und alter Bienen soll der Gehalt der Nassanov-Drüse an Geraniol (Boch und Shearer, 1963), das auf VarroaWeibchen stark repellierend wirkt, verantwortlich sein (Hoppe und Ritter, 1988). Diese Versuche wurden sämtlich unter Laborbedingungen durchgeführt, Experimente in Bienenvölkern standen noch aus. Ich habe daher dort den Verbleib frischgeschlüpfer Milbenweibchen untersucht, wobei ich im Herbst und damit unter drohnenfreien Bedingungen den Mil- 
benbesatz bei Arbeiterinnen vom Schlupf an verfolgt habe.

\section{MATERIAL UND METHODEN}

Anfang September wurden einem Volk (2zargig, DN) von Apis mellifera carnica der Tübinger Versuchsbienenhaltung zwei Waben mit verdeckelter Arbeiterinnenbrut entnommen und im Labor zum Schlupf gebracht. Alle binnen 24 $\mathrm{h}$ geschlüpften ca. 800 Jungbienen wurden auf dem Thorax mit einem Farbtupfen (ModellbauFarben, Fa Revell) markiert, wobei zwischen Varroa-Trägerinnen und milbenfreien Bienen unterschieden wurde (Tabelle I), und sogleich in das Volk zurückgesetzt. Anschließend wurde einen Monat lang kontrolliert, welche der markierten Bienen weiterhin Milbenträger waren. Außerdem wurde stichprobenweise der Infektionsgrad des gesamten Volkes ermittelt. Hierzu wurden von Waben mit viel offener Brut ungefähr 300 Bienen abgefegt, betäubt und die aufsitzenden Milben gezählt. Während des Versuchs hatte das Volk immer Brut, allerdings wurde das Brutnest kleiner.

\section{ERGEBNISSE}

Innerhalb eines Tages nach dem Schlupf wechselten fast $90 \%$ der frischgeschlüpften Milbenweibchen die Trägerbiene (Abb 1). Gleichzeitig wurden ungefähr $10 \%$ der zunächst als milbenfrei markierten Jungbienen befallen (Tabelle I). Genau in dieser Größenordnung lag gleichzeitig auch der mittlere Befallsgrad unmarkierter Bienen auf Brutwaben.

In der Folge nahm der Prozentsatz befallener Bienen zu. Bei den unmarkierten Bienen auf Brutwaben wurde nach 2 Wochen als höchste Befallsrate ein Mittelwert von etwas über $40 \%$ ermittelt. Von den markierten Bienen wurden an den Kontrollterminen zunächst über $30 \%$ und gegen Versuchsende noch $10-20 \%$ wieder beobachtet (Tabelle I), wobei von diesen 20-30\% Milben trugen (Abb 1). Die Gruppen-Durchschnittswerte der beim Schlupf zunächst als mit oder ohne Milben (Tabel-

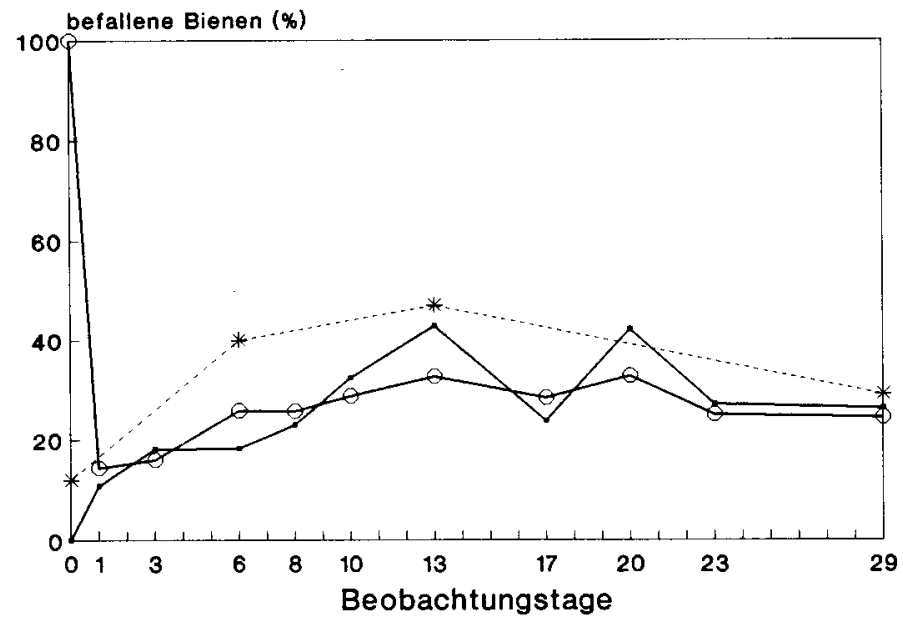

Abb 1. Veränderungen im Befallsgrad durch Varroa-Milben bei Arbeiterinnen eines drohnenfreien Herbstvolkes von Apis mellifera carnica. Unmittelbar nach dem Schlüpfen wurden je ca 400 Jungbienen mit $(\mathrm{O})$ bzw ohne $($.$) aufsitzende Milbenweibchen markiert. Außerdem wurden einige etwa eben-$ sogroße Stichproben von Stockbienen (*) untersucht. Unter der X-Achse sind die Beobachtungstermine angegeben. Die Milben verließen größtenteils die frisch geschlüpften Bienen, einen erhöhten Befallsgrad wiesen Arbeiterinnen ab dem 6 . Tag auf. 
e I) unterschiedenen Arbeiterinnen waren später stets sehr ähnlich (Abb I).

Um zu prüfen, ob auch im drohnenfreien Herbstvolk von den Milben Bienen bestimmter Alters- bzw. Funktionsgruppen bevorzugt werden, habe ich die markierten und daher altersbekannten Bienen nach normalem Ammenalter sowie als jünger bzw älter gruppiert und hierfür den mittleren Befall berechnet (Tabelle II). Einen deutlich niedrigeren Milbenbesatz wiesen nur die Jungbienen auf. Der durchschnittli-

Tabelle I. Milbenbesatz von Arbeiterinnen, die beim Schlüpfen Varroa-Weibchen trugen oder milbenfrei waren, im Verlauf von 4 Wochen in einem drohnenfreien Herbstvok von Apis mellifera carnica mit Brut.

\begin{tabular}{|c|c|c|c|c|c|c|c|}
\hline \multirow{2}{*}{\multicolumn{2}{|c|}{ Beobachtungszeit }} & \multicolumn{6}{|c|}{ Markierung } \\
\hline & & \multicolumn{3}{|c|}{ weiß } & \multicolumn{3}{|c|}{ rot } \\
\hline Tag & Datum & \multicolumn{2}{|c|}{ Milben } & $\begin{array}{l}\text { beobachtete } \\
\text { Bienen (\%) }\end{array}$ & & ohne & $\begin{array}{c}\text { beobachtete } \\
\text { Bienen (\%) }\end{array}$ \\
\hline 0 & 3 Sept & - & 416 & 100,0 & 381 & - & 100,0 \\
\hline 1 & 4 Sept & 20 & 166 & 44,7 & 21 & 126 & 38,6 \\
\hline 3 & 6 Sept & 27 & 122 & 35,8 & 24 & 127 & 39,6 \\
\hline 6 & 9 Sept & 25 & 112 & 32,9 & 32 & 92 & 32,5 \\
\hline 8 & 11 Sept & 33 & 111 & 34,6 & 27 & 78 & 27,5 \\
\hline 10 & 13 Sept & 43 & 90 & 32,0 & 29 & 72 & 26,5 \\
\hline 13 & 16 Sept & 42 & 98 & 33,7 & 32 & 66 & 25,7 \\
\hline 17 & 20 Sept & 24 & 77 & 24,3 & 17 & 43 & 15,7 \\
\hline 20 & 23 Sept & 32 & 44 & 18,3 & 18 & 37 & 14,4 \\
\hline 23 & 26 Sept & 13 & 35 & 11,5 & 8 & 24 & 8,4 \\
\hline 29 & $2 \mathrm{Okt}$ & 20 & 56 & 18,3 & 12 & 37 & 12,9 \\
\hline
\end{tabular}

Tabelle II. Durchnittlicher Milben-Befallsgrad während bestimmter Altersphasen bei Arbeiterinnen eines brütenden Herbstvokes, die beim Schlupf als Milbenträger oder milbenfrei markierten worden waren. Der allgemeine Status bezieht sich auf den normalen Tätigkeitskalender, der hier nicht überprüft wurde. Ein relative hoher Befall wurde ab dem 6 . Imaginaltag festgestellt. $\bar{x}=$ Mittelwert, $s=$ Standardabweichung, nach Beobachtungsdaten von Tabelle I.

\begin{tabular}{|c|c|c|c|c|c|}
\hline \multirow{4}{*}{ Allgemeiner Status } & \multirow{4}{*}{ Alter (d) } & \multicolumn{4}{|c|}{ Markierung } \\
\hline & & \multicolumn{2}{|c|}{ weiß } & \multicolumn{2}{|c|}{ rot } \\
\hline & & \multicolumn{4}{|c|}{ Arbeiterinnen mit Milben (\%) } \\
\hline & & $\bar{x}$ & s & $\bar{x}$ & $s$ \\
\hline Frischgeschlüpfe & 0 & 0,0 & & 100,0 & \\
\hline Jungbienen & $1-3$ & 14,4 & 3,8 & 15,1 & 3,9 \\
\hline Ammen & $6-13$ & 29,9 & 5,4 & 28,2 & 5,3 \\
\hline Altbienen & $17-29$ & 29,8 & 5,5 & 27,7 & 5,3 \\
\hline
\end{tabular}


che Infektionsgrad von Bienen im Ammenalter und danach unterschied sich dagegen nicht, er lag einheitlich bei $30 \%$.

Der allgemeine Befallsgrad des Versuchsvolkes entsprach im übrigem dem anderer Völker im Tübinger Bienengarten während des Rückgangs des Brutgeschäftes im Verlauf des Septembers bis Anfang Oktober (Tabelle I).

\section{DISKUSSION}

Im Herbstvolk nimmt bei rückläufigem Brutgeschäft der Anteil an VarroaWeibchen, die sich in der phoretischen Phase befinden, rasch zu, bis er im Wintervolk $100 \%$ erreicht. Einen deutlichen Anstieg im Befallsgrad der adulten Arbeiterinnen des Versuchsvolkers konnte ich während der ersten beiden Wochen des Beobachtungszeitraumes zwar registrieren (Abb 1), es war jedoch stets offene Brut vorhanden, so daß die Milbenweibchen invadieren konnten. Auch Ammenbienen müssen daher in Funktion gewesen sein.

Die Chance, in eine kurz vor der Verdeckelung stehende Brutzelle einzudringen, ist am höchsten, wenn sich die Milben auf Ammenbienen aufhalten. Meine Befallsdaten der markierten Bienen zeigen klar, daß über $6 \mathrm{~d}$ alte Arbeiterinnen wesentlich häufiger Milben tragen als jüngere (Tabelle II). Allerdings war kein Unterschied im Befall sämtlicher bis zu ungefähr einen Monat alten Bienen festzustellen. Dies dürtte mit dem Übergang zum Winterbienen-Status zusammenhängen.

Ein frischgeschlüpftes Milbenweibchen kann entweder auf derjenigen Biene, mit der es die Brutzelle verlassen hat, sitzen bleiben, bis diese zur Amme wird. Oder die Milbe kann die Trägerbiene mit dem Ziel wechseln, auf eine Amme umzusteigen. Meine Ergebnisse belegen, daß die
Varroa-Weibchen offensichtlich die letztgenannte Strategie verfolgen. Sie dürtte bei stärkerer Parasitierung zudem eine bessere Gleichverteilung der Milben im Volk bewirken und außerdem verhindern, daß mehrere Milbenweibchen auf einer Biene verbleiben. Auch dies könnte die Invasionschancen mindern. Insgesamt bestätigen meine in einem starken, drohnenfreien Volk erhobenen Daten die bislang nur auf Laborversuchen beruhenden Vorstellungen über eine Bevorzugung zwar junger, nicht aber frisch geschlüpfter Stockbienen durch die Milbenweibchen.

Es bleibt die Frage nach den Mechanismen, die ein solches Verhalten der Milben steuern. In meinem Herbstvolk war das zusätzliche Problem einer Diskriminierung von Arbeiterinnen und Drohnen nicht mehr gegeben. Konkret stellt sich die Frage, anhand welcher Parameter eine Milbe das Alter einer Biene erkennen kann. Vieles spricht für das Pheromon-Bukett des Wirtsindividuums. Alterskorrelierte quantitative Veränderungen wurden zB für das Muster volatiler Kohlenwasserstoffe des Stachelapparates beschrieben (McDaniel et al, 1987). In Olfaktometer-Tests stellten Kraus et al (1986) ebenso wie Rosenkranz (1990) generell eine erhöhte Attraktivität von Ammen bzw. Stockbienen für VarroaWeibchen fest. Ob die Milben bei dieser Wirtswahl die gleichen Reizmuster verwerten wie bei der Entscheidung für eine alte Bienenlarve (Le Conte et al, 1990), bleibt zu klären.

\section{DANKSAGUNG}

Dem DAAD danke ich für ein Promotionsstipendium und CAPES für Übernahme von Reisekosten.

Summary - Distribution of Varroa ja-
cobsoni within a drone-free honey bee 
colony (Apis mellifera carnica). In an autumn colony $\approx 800$ newly emerged workers were checked for carrying mites, marked properly, and reintroduced into the hive. Then over a period of $30 \mathrm{~d}$ the marked bees were controlled every few days for mite infestation (table I). It was observed that within a short time after emergence, most Varroa females switched their carrier host bees (fig 1). Of the workers up to $3 \mathrm{~d}$ old $\approx 15 \%$ were observed with mites, and after $d 6$ this infestation increased to $\approx 30 \%$. There was no difference between bees which at emergence were carrying mites or not. Since no drones were present, the female mites in choosing their carrier bee evidently considered only the age of the workers. In order to optimize their chance to invade a brood cell prior to operculation, the mites may prefer nurse bees. In this experiment there was no difference in infestation of workers beyond nursing age, probably because of the switch to winter conditions. The average mite infestation of unmarked workers sampled from brood frames was nearly the same as in the marked bees. The distribution of mites reported here for a colony is in agreement with data obtained in laboratory experiments. According to the literature, the behavior of Varroa females in selecting optimal host stages presumably depends on the pheromone bouquet of the individual bee.

\section{Varroa jacobsoni / Apis mellifera carni- $\mathrm{ca} /$ parasite behaviour / host selection}

\section{Résumé - Répartition de Varroa jacob-} soni au sein d'une colonie d'abeilles (Apis $m$ carnica) dépourvue de mâles. On a vérifié la présence/absence de l'acarien Varroa jacobsoni sur environ 800 abeilles récemment écloses et prélevées dans une colonie à l'automne. Les abeilles ont été marquées, puis réintroduites dans la ruche. L'infestation par les acariens des abeilles marquées a été régulièrement contrôlée pendant $30 \mathrm{j}$ (tableau I). Durant une courte période suivant l'émergence la plupart des femelles de Varroa changent d'abeille hôte (fig 1). Environ 15\% des ouvrières âgées de 1 à $3 \mathrm{j}$ sont porteuses d'acariens et après 6 j l'infestation atteint environ $30 \%$. II n'y a pas de différence entre les abeilles infestées à l'émergence et celles qui ne l'étaient pas. Puisqu'il n'y a pas de mâles, les acariens femelles ne prennent en compte que l'âge des ouvrières lors du choix de l'abeille comme vecteur. Pour optimiser les chances d'envahir une cellule de couvain avant l'operculation, les acariens femelles devraient préférer les nourrices. Dans notre expérience il n'y a pas de différence dans l'infestation des ouvrières de classe d'âge supérieure à celle des nourrices, probablement à cause du passage aux conditions hivernales. L'infestation moyenne des ouvrières non marquées prélevées sur les rayons de couvain est presque samblable à celle des ouvrières marquées. La répartion des acariens décrite ici au sein d'une colonie est en accord avec les données obtenues par les expériences faites en laboratoire. Selon la littérature, le comportement des femelles de Varroa lors du choix du stade optimal de l'hôte dépend vraisemblablement du bouquet phéromonal de l'ouvrière considérée.

Varroa jacobsoni / choix de l'hôte / comportement parasite

\section{LITERATUR}

Boch R, Shearer DA (1963) Production of geraniol by honeybees of various ages. $J$ insect Physiol 9, 431-434

Hoppe H, Ritter W (1988) The influence of the Nasonov pheromone on the recognition of house bees and foragers by Varroa jacobsoni. Apidologie 19, 165-172 
Ifantidis M, Rosenkranz $P$ (1988) Reproduktion der Bienenmilbe Varroa jacobsoni (Acarina: Varroidae). Entomol General 14, 111-122

Kraus B, Koeniger N, Fuchs S (1986) Unterscheidung zwischen Bienen verschiedenen Alters durch Varroa jacobsoni Oud, und Bevorzugung von Ammenbienen im Sommerbienenvolk. Apidologie 17, 257-266

Le Conte $Y$, Arnold $G$ (1987) Influence de l'âge des abeilles (Apis mellifera $L$ ) et de la chaleur sur le comportement de Varroa jacobsoni Oud. Apidologie 18, 305-320

Le Conte $Y$, Arnold G, Trouiller J, Masson C, Chappe B (1990) Identification of a brood pheromone in honeybees. Naturwissenschaften 77, 334-336

McDaniel CA, Howard AM, Collins, Brown WA (1987) Variation in the hydrocarbon composition of non-africanized Apis mellifera $L$ sting apparatus. Sociobiology 13, 133-143

Ritter W (1981) Varroa disease of the honey bee Apis mellifera. Bee World 62, 141-153

Rosenkranz P (1990) Wirtsfaktoren in der Steuerung der Reproduktion der parasitischen Bienenmilbe Varroa jacobsoni in Völkern von Apis mellifera. Dissertation Fak Biol Univ Tübingen 